\title{
ALLOPURINOL IN PATIENTS WITH IMPAIRED RENAL FUNCTION
}

\author{
BY \\ N. W. LEVIN AND O. L. ABRAHAMS \\ From the C.S.I.R. Cardio-Pulmonary Unit, Department of Medicine, University of the Witwatersrand, \\ and the Renal Unit, Johannesburg General Hospital, Republic of South Africa
}

The xanthine oxidase inhibitor allopurinol would appear to be ideally suited for the treatment of patients with recurrent urinary uric acid stones or gout with impaired renal function. The latter group are often unresponsive to uricosuric agents and before the advent of allopurinol no suitable therapy was available. The present study was carried out in order to answer the following questions:

(1) Is allopurinol as successful in decreasing serum uric acid (SUA) excretion in patients with renal disease as it is in individuals with normal renal function?

(2) Do larger doses have to be used in the treatment of patients with impaired renal function to obtain satisfactory clinical response?

(3) Does allopurinol therapy have any direct or indirect effect on renal function?

(4) Are the side-effects of allopurinol therapy different in patients with impaired renal function?

(5) Is the incidence of urinary calculi decreased in patients with impaired renal function while on allopurinol therapy?

(6) Is the increased oxypurine clearance noticed in normal patients on allopurinol therapy also observed in patients with renal functional impairment?

(7) What is the effect of urinary alkalinization on oxypurine excretion in patients on allopurinol therapy?

\section{Material and Methods}

Of 33 patients studied ( 29 men and 4 women, aged from 8 to 68 years), 22 had endogenous creatinine clearance less than and eleven more than $70 \mathrm{ml} . / \mathrm{min}$. Twelve patients had a recent history of urinary uric acid calculi and four had gross tophaceous gout.

After a control period of 10 days, when all treatment was discontinued, measurements were made of endogenous creatinine clearance, uric acid clearance, urea clearance, and urinary protein excretion.

In twelve patients standard phenolsulpthalein (PSP) excretions were measured. Urine was examined microscopically. Serum iron and iron-binding capacity estimations, SGPT measurements, and full blood counts were also carried out. After starting allopurinol therapy 300 to $400 \mathrm{mg}$./day, repeated evaluation of renal function and measurements of urinary and serum urate levels were carried out. Full blood counts were performed twice monthly and in the majority of patients repeated estimations of SGPT and SGOT were also done. Patients were seen on an average of every 10 days to 2 weeks and a careful history was taken of attacks of acute gout and of episodes of urinary calculi. Of the 33 patients, all were followed for at least 3 months, 21 for 6 months, 13 for 9 months, and 9 for 12 months. Uric acid was measured on some patients using the Autoanalyser cyanide method. Creatinine was measured by the alkaline picrate method, using Lloyd's reagent. Io some patients measurements were made of urate an oxypurine clearances before and after allopurinol, an in four patients (two in renal failure and two wit moderate impairment of renal function) the effect alkalinization of the urine on oxypurine and uric acid clearance was observed. In these studies plasma and urinary uric acid concentrations were measured by ultraviolet spectrophotometry, using uricase [4 units of Seravac Laboratories (Pty) Ltd. uricase] according to the method of Praetorius and Poulsen (1953). Heparinized plasma, separated immediately after collection of the blood, was used in preference to serum, as oxypurines were estimated on the same sample and the breakdown of adenosinetriphosphate (ATP) contained in the blood leads to formation of hypoxanthine (Jørgensen and Poulsen, 1955b).

Oxypurines were estimated in heparinized plasma and in urine by the method of Watts, Watkins, Mathias, and Gibbs (1966). The recovery of xanthine added to plasma and urine using this method was 90 to 95 per cent. It was found that the presence of 1 to $40 \mu \mathrm{g}$. 4, 6-dihydroxypyrazolopyrimidine (DHPP, allo-xanthine) in the spectrophotometer quartz curvette did not inhibit the conversion of $1 \mu \mathrm{g}$. xanthine to uric acid using $0.02 \mathrm{ml}$. of a 10-fold dilution of xanthine oxidase (xanthine oxidase, 40 units, Nutritional Biochemicals Corporation).

Plasma levels of DHPP were estimated as described by Rundles, Metz, and Silberman (1966b). It was found that the addition of xanthine or hypoxanthine in concentrations of $\mathbf{4 0}$ per cent. or less of the DHPP concentration had no effect on the inhibitory properties of DHPP under the experimental conditions, but DHPP concentration 
could not be reliably estimated when the concentration of oxypurines present exceeded 40 per cent. of the concentration of DHPP.

The solubility of xanthine, hypoxanthine, uric acid, and DHPP was measured in both plasma and urine (the latter at $p \mathrm{H} 5$ and 8 ). The respective media were incubated for $2 \mathrm{hrs}$ at $37^{\circ} \mathrm{C}$. with each of the purines, mixing well at 10-min. intervals. At the end of the incubation period, the mixture was filtered and the filtrate tested. Untreated urine and plasma were tested at the same time.

\section{Results}

(1) Acute Attacks of Gout.-There was a marked reduction in the number of acute attacks of gout in all the patients who were subject to this complication.

There did not seem to be an increased rate of exacerbation of acute gouty arthritis in the early stages of treatment with the doses used. All patients were given colchicine $1 \mathrm{mg}$. twice a day for at least the first 2 months because of the reported high incidence of acute gouty arthritis in patients receiving allopurinol. The acute attacks of gout which occurred seemed of much decreased severity. Many patients commented that numerous aches and pains that they had previously noticed in the muscles of their back, shoulders, and arms seem to have disappeared on allopurinol therapy. Limitation of mobility often improved remarkably. Despite these favourable findings, occasional acute attacks of gout did occur in patients with SUA levels well below $5 \mathrm{mg} . / 100 \mathrm{ml}$. There seemed to be no difference in the favourable response of patients with greater impairment of renal function as compared to the others.

(2) Incidence of Calculi.- Of the twelve patients who had a history of urinary calculi or gravel, only two had any such symptoms during the trial (Table I).

One was Case 8, who passed two uric acid stones at a
TABLE I

EFFECTS OF ALLOPURINOL ON INCIDENCE OF URINARY CALCULI

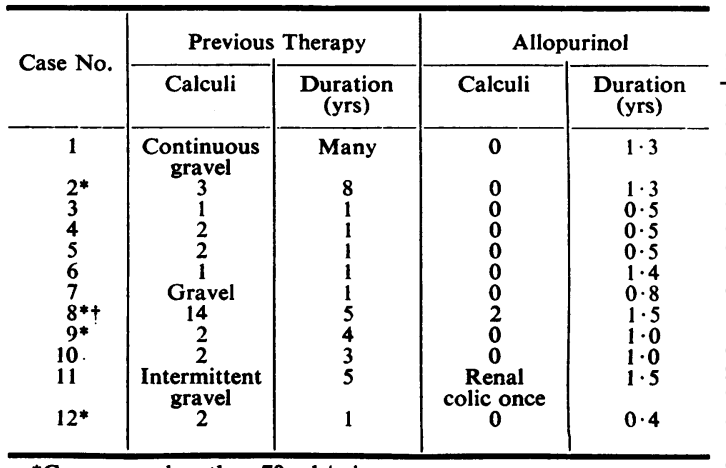

* $\mathrm{C}_{\text {creatinine }}$ less than $70 \mathrm{ml}$. $/ \mathrm{min}$.

†Uric acid stones passed while urinary urate excretion $110 \mathrm{mg}$./day.

time when his urinary urate excretion was less than 120 음 $\mathrm{mg}$./day. It appeared that his urine dissolved less 9 xanthine than that of patients with a similar degree of $\infty$ impairment of renal function (see Table II). The

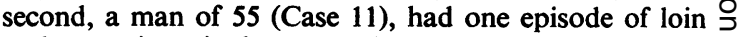
and ureteric pain but no calculus was passed and an intravenous pyelogram carried out a few days later $z$ showed no abnormality.

Many patients who had previously had a persiste $\stackrel{\Phi}{\stackrel{\oplus}{~}}$ history of passing urinary gravel on mild dehydray tion were completely symptom-free on allopurine therapy.

(3) Tophi.-In four patients with gross tophace ous gout treated for more than 9 months each, there was softening and reduction in the size of tophi leading to complete disappearance of some of them. Renal function was impaired in these patients ( $\mathrm{C}_{\text {creatinine }} 48,63,96,82 \mathrm{ml} . / \mathrm{min}$.).

(4) SUA Levels.-The effect of allopurinol on $\frac{0}{3}$ SUA levels and the mean dosage used during 3,6,9, and 12 months of therapy are shown in Fig. 1 \% (opposite). The mean reduction in SUA level in

TABLE II

SOLUBILITY OF PURINES AND THE PYRIMIDINE DHPP IN PLASMA AND URINE

\begin{tabular}{|c|c|c|c|c|c|c|c|c|c|c|c|c|}
\hline \multirow{2}{*}{$\begin{array}{l}\text { Purine or Pyrimidine } \\
\text { (mg./100 ml.) }\end{array}$} & & \multirow[t]{2}{*}{$\cdots$} & \multicolumn{2}{|c|}{ Uric Acid } & \multicolumn{4}{|c|}{ Xanthine } & \multicolumn{3}{|c|}{ Hypoxanthine } & \multirow{2}{*}{$\begin{array}{c}\text { DHPP } \\
\text { (1) }\end{array}$} \\
\hline & & & (1) & (4) & (1) & (2) & (3) & (4) & (1) & (2) & (4) & \\
\hline Plasma & & & & 7 & 20 & 20 & & 10 & & & 115 & $20-30$ \\
\hline Urine & $\begin{array}{l}p \mathrm{H} \\
p \mathrm{H}\end{array}$ & & $\begin{array}{l}15 \\
93\end{array}$ & & $\begin{array}{l}12 \\
48\end{array}$ & $1-2$ & $\begin{array}{r}3 \\
17\end{array}$ & & $\begin{array}{l}120 \\
110\end{array}$ & $\begin{array}{l}120 \\
105\end{array}$ & & $\begin{array}{l}20 \\
70\end{array}$ \\
\hline
\end{tabular}

(1) Normal subiect.

(2) Patient who developed uric acid calculi (Table I, Case 8) while on allopurinol ( $\mathrm{C}_{\text {creatinine }} 12 \mathrm{ml}$./min.).

(3) Patient with gouty nephropathy $\left(C_{\text {creatinine }} 10 \mathrm{ml} . / \mathrm{min}\right.$.) (Table $I$, Case 11$)$.

(4) Measurements reported by Klinenberg and others (1965). 


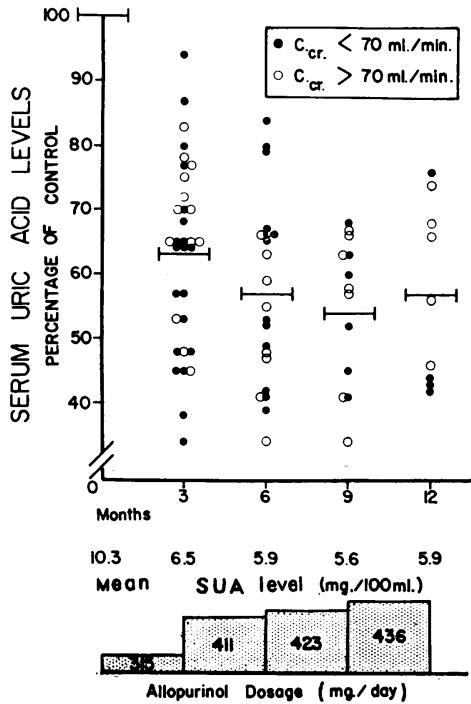

Fig. 1.-Effect of allopurinol therapy on serum uric acid levels.

those with renal impairment does not differ from that in those with normal renal function. There is no significant difference in the dosages used in these two groups.

(5) Urinary Urate Excretion.-The effect of allopurinol during 3, 6, 9, and 12 months as compared to control levels is shown in Fig. 2. Again there is no significant difference between patients

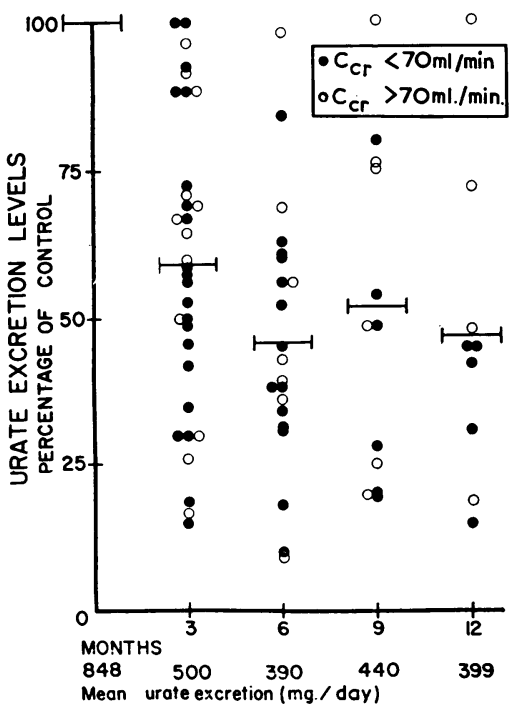

Fig. 2.-Effect of allopurinol therapy on urinary urate excretion with renal impairment and those with normal renal function. Although a substantial decrease in urinary urate excretion is demonstrated, the doses used were not large and it is very likely that with larger doses a greater reduction in excretion would have been produced. The greater decrease in urate excretion after the first 3 months can be attributed to the slightly higher dosages used after that time.

(6) Renal Function.-Fig. 3 shows the mean endogenous creatinine clearance at the beginning and end of the study in 29 of the 33 patients. In the other four patients clearances were 51, 60,56, and 64 $\mathrm{ml}$./min. initially and $70,67,64$, and $63 \mathrm{ml} . / \mathrm{min}$. at $5,5,12$, and 4 months later respectively. There are no significant changes in $\mathrm{C}_{\text {creatinine. }}$ Certainly those patients with impairment of renal function showed no consistent deterioration.

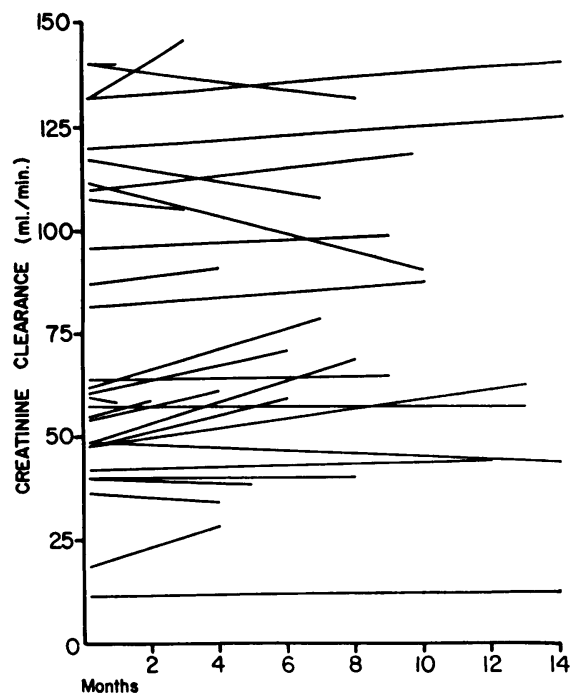

Fig. 3.-Effect of allopurinol therapy on endogenous creatinine clearance.

Fig. 4 (overleaf) shows no significant change in the percentage blood urea level. Thus it can be concluded that there was no significant change in renal function as measured by these parameters. There was no significant change in urinary protein excretion and twelve patients in whom PSP excretion was measured showed no significant change. Microscopic examination of the urine also showed no change except for disappearance of haematuria in some patients who had stones. (The precise reason for the impairment of renal function in the patients in this trial was not established by renal biopsy. However, from the clinical findings it would seem 


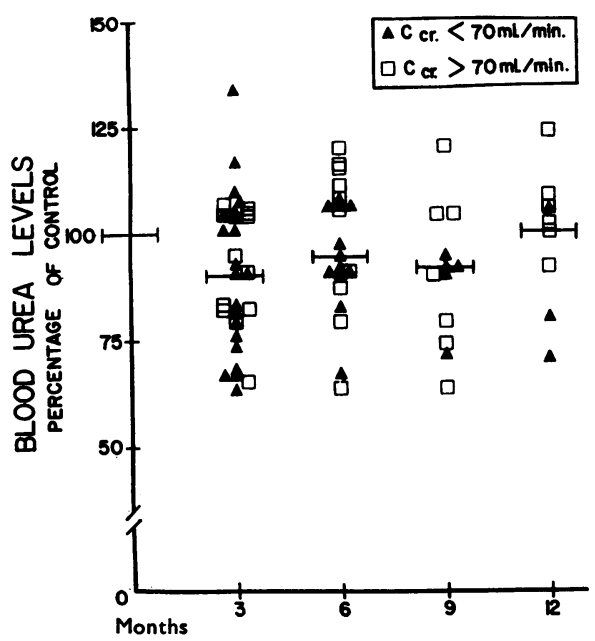

Fig. 4.-Percentage change in blood urea level during allopurinol therapy.

that the only important factor in this group was the presence of gout. The effects of chronic infection following stones cannot be excluded).

(7) Oxypurine/Uric acid Excretion.-The relationship of $\frac{\mathrm{C}_{\text {oxypurine }}}{\mathrm{C}_{\text {urate }}}$ to creatinine clearance is shown in Fig. 5.

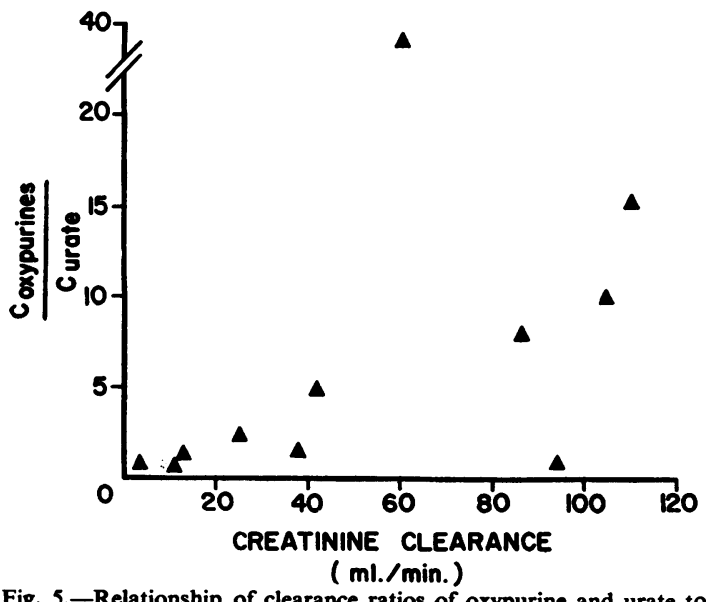

Fig. 5.-Relationship of clearance ratios of oxypurine and urate to endogenous creatinine clearance.

The ratio tends to be higher in the patients with better renal function. Uric acid clearance is much less affected by the state of renal function. Fig. 6 shows the expected effect of allopurinol therapy on the $\frac{\text { oxypurine }}{\text { urate }}$ excretion ratio. Except for one

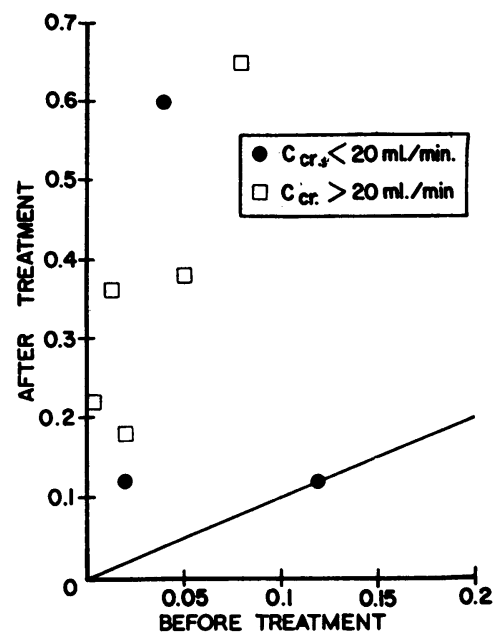

Fig. 6.-Effect of allopurinol therapy on $\frac{\text { oxypurine }}{\text { urate }}$ excretion ratio in patients with creatinine clearances less than and greater than 20 $\mathrm{ml} . / \mathrm{min}$.

Clearances $51,94,58,48$, and $118 \mathrm{ml} . / \mathrm{min}$.

Clearances $11,8,6 \mathrm{ml} . / \mathrm{min}$., both reading from left to right.

patient with a creatinine clearance of $8 \mathrm{ml} / \mathrm{min}$ there was a substantial increase in the ratio. However, in two uraemic individuals, the $\frac{\mathrm{C}_{\text {oxypurine }}}{\mathrm{C}_{\text {urate }}}$ ratios did not increase (Fig. 7), while patients with less impairment of renal function showed an in $\vec{\varphi}$ creased ratio. The absence of increase in oxypurite clearance in patients in renal failure taking allo ${ }^{+}$

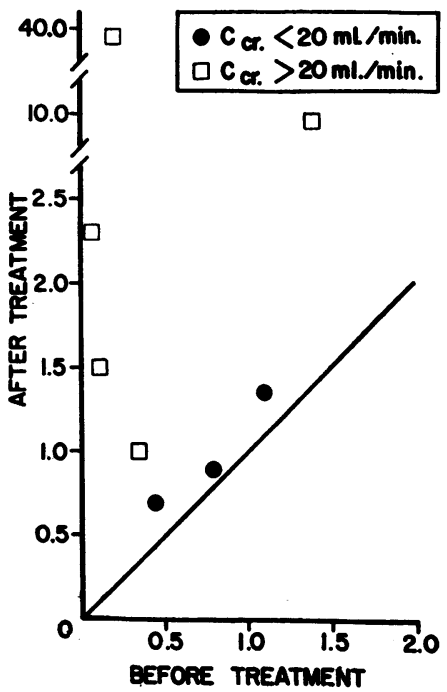

Fig. 7.-Effect of allopurinol therapy on $\frac{C_{\text {oxypurine }}}{C_{\text {urate }}}$ ratio.

Clearances $51,58,48,118$, and $94 \mathrm{ml} . / \mathrm{min}$.

Clearances 6,8 , and $11 \mathrm{ml}$./min., both reading from left to right 
purinol was not due to a decrease in urinary excretion so much as to retention of plasma oxypurines.

The previously reported disparity between uric acid excretion before and the sum of uric acid and oxypurine excretion after allopurinol therapy was observed in this study. In seven subjects with renal impairment mean 24-hour urate excretion before therapy was $688 \mathrm{mg}$. while total purine excretion (uric acid and oxypurine) after therapy was $576 \mathrm{mg}$.

(8) Effect of Urinary Alkalinization on Oxypurine Clearance.-Oxypurine excretion and clearances increased in two patients with $\mathrm{C}_{\text {creatinine }}$ of 48 and $120 \mathrm{ml} . / \mathrm{min}$. but not in those with $\mathrm{C}_{\text {creatinine }}$ of 10 and $8 \mathrm{ml} . / \mathrm{min}$. (Fig. 8). Increase in urinary $p \mathrm{H}$ was associated with an increase in urine flow.

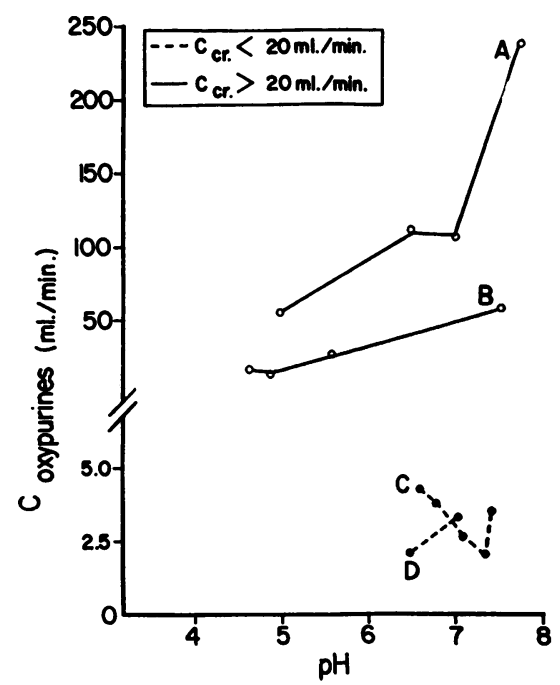

Fig. 8.-Effect of urinary alkalinization ( $p \mathrm{H}$ increase) on oxypurine clearance during allopurinol therapy.

Urine Flow $\left\{\begin{array}{l}\mathrm{A} \quad 0.8 ; 2.0 ; 3.8 ; 5.2 \\ \mathrm{~B} 0.8 ; 1.2 ; 1.6 ; 2.1\end{array}\right.$

(ml./min.) $\begin{cases}\text { C } & 1.9 ; 2.4 ; 3.6 ; 4.7 ; 4.5 \\ D & 1.0 ; 1.1\end{cases}$

TABLE III

PLASMA DHPP LEVELS AND CREATININE CLEARANCE IN PATIENTS ON ALLOPURINOL THERAPY

\begin{tabular}{c|c|c}
\hline Case No. & $\begin{array}{c}\text { DHPP } \\
\text { (mg./100 ml.) }\end{array}$ & $\begin{array}{c}\text { C }_{\text {creatinine }} \\
\text { (ml./min.) }\end{array}$ \\
\cline { 2 - 3 } & 0.8 & 140 \\
2 & 1.5 & 58 \\
3 & 0.6 & 63 \\
4 & -51 \\
5 & 1.2 & 118 \\
6 & 0.8 & 94 \\
7 & -121 \\
8 & 0.8 & 48 \\
9 & 1.0 & 63 \\
10 & 3.0 & 8 \\
11 & 2.3 & 11 \\
12 & & 10 \\
\hline
\end{tabular}

(9) Plasma Levels of DHPP (Table III).-Some of the values found are not true measures of con- क centrations as the plasma oxypurine concentration $\overrightarrow{\vec{F}}$ was more than 40 per cent. of the DHPP concentration found. In these cases the levels of DHPP found are less than the true concentration. There was a tendency for the DHPP concentration to be $\vec{\nabla}$ higher in cases of renal failure.

(10) Side-effects of Therapy. - Two patients developed a maculopapular rash which disappeared $\overrightarrow{\vec{\omega}}$ immediately after cessation of therapy. In one patient this reappeared after therapy was re-institu- $\frac{\varrho}{2}$ ted and became worse with increasing dosage, while in the other patient it did not recur. Both patients of had impaired renal function, $\mathrm{C}_{\text {creatinine }}$ being 11 and $33 \mathrm{ml} . / \mathrm{min}$. respectively. Gastrointestinal upsets were not obvious during allopurinol therapy except $\left.\right|_{0}$ for persistent nausea in one patient.

The effects of allopurinol on iron absorption will $\stackrel{\infty}{\rightarrow}$ be the subject of a separate discussion, but in brief two techniques were utilized in order to measure this. -

One was the method of Fielding* (1965) for $Z$ measuring chelatable iron after the injection of $\overleftarrow{\leftarrow}$ desferrioxamine and labelled ferrioxamine (Fielding and Brunström, 1964). This was carried out i̊ $\mathbb{D}$ eight gouty patients, four who had been on allopure nol for at least 6 months and four who had not bee treated. The second technique was the double isotope method for iron absorption using labelled ferric chloride. This was carried out in a group of ten patients. There was no significant difference in these studies between the patients on allopurinol therapy and the untreated patients. Further studies on the effect of allopurinol on iron metabolism are in preparation for publication.

SGPT levels did not rise appreciably during the trial in 22 patients tested:

$\begin{array}{lcc}\text { SGPT (units) } & \text { Mean } & \text { Range } \\ \text { Control } & 18 & 10-33 \\ \text { On allopurinol } & 21 & 12-31\end{array}$

There were occasional increases of SGPT above the limit of normal but these returned to normal 을 without discontinuing allopurinol.

(11) Solubility.-The solubility of xanthine, $\bar{N}$ hypoxanthine, uric acid, and DHPP was measured in both serum and urine, the latter at $p H 5$ and 8 (Table II).

*These studies were carried out by Dr. P. Jacobs and Dr. R. Green under the direction of Dr. T. H. Bothwell. 


\section{Discussion}

Previous studies (Yü and Gutman, 1964; Klinenbarg, Goldfinger, and Seegmiller, 1965) have shown that allopurinol is of considerable value in the management of gouty patients, particularly those with a tendency to stone formation. This study confirms previous results and extends it also to those with impaired renal function. The results do not indicate that a larger dose of allopurinol is necessary in patients with markedly impaired kidney function. The incidence of side-effects does not appear to be any higher in those who have decreased glomerular filtration rates.

Although xanthine is less soluble in urine than uric acid it is more soluble in serum. A theoretical hazard of allopurinol therapy is the formation of xanthine stones as has been demonstrated to occur in congenital xanthinuria. However, this has not yet been reported during allopurinol therapy. The clearance of oxypurines is much greater than that of uric acid during allopurinol therapy (Goldfinger, Klinenberg, and Seegmiller, 1965) and it was thought important to ascertain whether in renal failure the renal handling of these substances was similar. The present studies show an increased rate of oxypurine excretion in all patients treated including those with marked renal impairment. However, oxypurine clearance did not increase to the same extent in the latter group because of the tendency of these patients to retain oxypurines. The highest plasma oxypurine level reached was $1.9 \mathrm{mg} . / 100 \mathrm{ml}$. in a patient with terminal renal failure given $600 \mathrm{ml}$. allopurinol daily; thus although xanthine is soluble in plasma to the extent of $20 \mathrm{mg}$. $/ 100 \mathrm{ml}$., the chances of xanthine gout occurring would appear to be remote even in renal failure. DHPP plasma levels also tended to be higher in patients with impaired renal function (Table III), but the level reached was far below the saturation level. The likelihood of allopurinol gout is therefore negligible. It is perhaps fortunate that the relative solubilities of xanthine and uric acid are such that retention of xanthine in renal failure in patients on allopurinol is not disadvantageous.

The patients studied in this series who were given allopurinol were told to alkalinize their urine in view of the decreased solubility of uric acid and xanthine in acid urine. The present preliminary studies have shown that alkalinization of the urine is attended by an increased clearance of these substances. It would appear that to some extent the excretion of these substances is influenced by the $p H$ of the urine. Xanthine has pKs of $0 \cdot 8,7 \cdot 44$, and
$11 \cdot 1$, and hypoxanthine pKs of $1 \cdot 98,8 \cdot 94$, and $12 \cdot 10$, but the oxypurines are not lipid-soluble and it is therefore unlikely that non-ionic diffusion plays an important part in their handling by the kidney. Increased excretion with urinary alkalinization was more obvious in those patients with normal renal function, and if the assumption is made that half the oxypurine excretion is xanthine the concentration of xanthine in the urine may conceivably approach saturation level under these circumstances. The rate of excretion is also influenced by the rate of urine flow in that a diuresis is accompanied by an increase in oxypurine excretion. The effects of urine flow and $p \mathrm{H}$ change have still to be separated and the effect of acetazoleamide has yet to be determined.

This study and others have demonstrated that the solubility of the oxypurines is influenced by the total quantity of purines present in the urine. Thus patients with low UUA concentrations will tend to have decreased solubility of oxypurines as compared to those with a high UUA concentration. This was well demonstrated by Case 8 (see Table I, above) who passed urate stones, perhaps coincidentally, while excreting very low quantities of urate [Table II (2)]. It was interesting that the solubility of xanthine in his urine was less than that of a patien with equally low uric acid excretion but no historg of stones [Table II (3)].

In the absence of a suitable control series it is difficult to assess the effects of allopurinol on rena function. The present trial shows no change in renal function during the study. A longer follow-up is necessary to be certain that allopurinol therapy does not eventually result in an improvement in renal function. In Case 8 there had been a gradual reduction in endogenous $C_{\text {creatinine }}$ until allopurinol therapy was started, and in the last 15 months while taking this drug there has been no further change, the endogenous $C_{\text {creatinine }}$ remaining at \pm 11 $\mathrm{ml} . / \mathrm{min}$. On theoretical grounds it would be expected that with a reduction in SUA and UUA concentration the possible deleterious effects of this substance on the kidney might be prevented. Certainly the marked reduction in the number of attacks of renal calculi would favourably influence renal function.

Preliminary observations on the effects of allopurinol on iron absorption would indicate that there is possibly some increased absorption of iron with the use of this drug. The quantitative aspects of this have yet to be determined but do not appear significant. It might, however, preclude the use of allopurinol in patients with iron overload of any cause. In regard to the side-effects of allopurinol, 
no consistent increase in SGPT have been demonstrated and no other change in hepatic function has been observed. The appearance of a maculopapular rash has been previously described in the literature and in the two instances observed in this trial the rash disappeared immediately after cessation of therapy. Apart from this there were no side-effects at all, and the drug would appear to be safe when used under the conditions of this trial. No change in any of the formed elements of the blood was observed on repeated testing throughout the course of the trial.

\section{Summary}

(1) Allopurinol was used successfully in patients with impaired renal function to reduce the incidence of urinary calculi and the frequency of acute attacks of gout.

(2) The same dosage scheme could be used in patients with renal impairment as in patients with normal renal function.

(3) No change in endogenous $C_{\text {creatinine, }}$ PSP excretion, or urinary protein excretion could be observed during the trial (33 patients $\pm 7,000$ patient days).
(4) Side-effects consisted of a maculopapular rash in two patients, which recurred in one on resuming therapy.

(5) The increased oxypurine excretion notedo during allopurinol therapy was observed in all등 patients tested including those with impaired renal $\overline{\overline{\bar{c}}}$ function, but oxypurine clearance did not increase $\widehat{\Phi}$ to the same extent in the latter group because of the increase in plasma oxypurines. The oxypurine ${ }^{\infty}$ levels observed did not approach saturation levels in $\overrightarrow{0}$ plasma but could possibly do so in urine. There $\vec{A}$ appeared to be an increase in oxypurine excretion in ${ }_{\sigma}^{\omega}$ two patients with good renal function coincident with increased urinary alkalinization. However, this was accompanied by a diuresis and the twoor factors were not separated.

Gratitude is expressed to Dr. B. Wallace and Dr. W, M. Politzer, Biochemistry Department, South African? Institute for Medical Research, to Mrs. S. M. Perold, $\infty$ Department of Chemical Pathology, University of the Witwatersrand, and to Dr. B. M. Bloomberg, $\frac{\text { S }}{2}$ Clinical Laboratories, Johannesburg, for biochemicalassistance, to Professor H. B. Stein for making facilities $Z$ available in his Department, and to Mrs. N. Hardie and? the Photographic Unit, Department of MedicinE, University of the Witwatersrand, for the illustrations. 